\title{
|||||||||||||||||||||||||||||||||||||||||||||||||||||||||||||||||||.
}
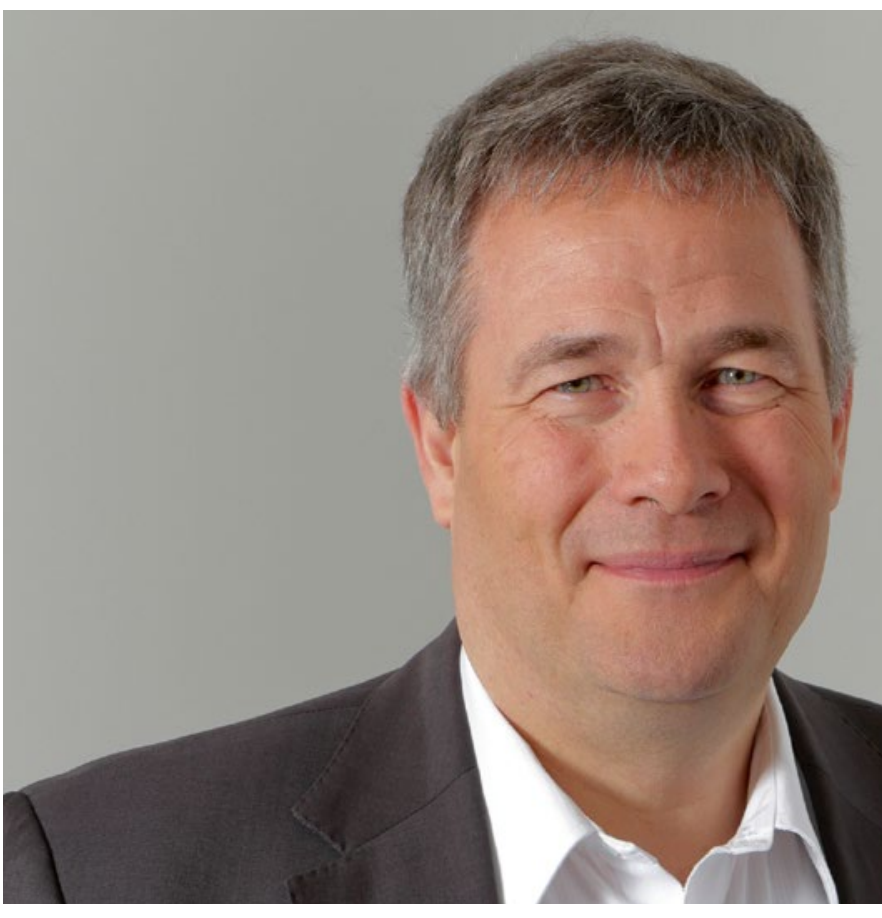

\section{Technik für Menschen}

Wenn ich mich noch einmal entscheiden müsste, welchen Beruf ich wähle, ich würde wieder Ingenieur werden. Ich liebe diesen Beruf und die Technik. So wie mir geht es vielen Ingenieurinnen und Ingenieuren. Aufgrund der Tatsache, dass die Anzahl der neuen Technologien, über die wir verfügen können, und der neuen Methoden, die wir einsetzen können, in den letzten Jahren stark zugenommen hat, wird das Betätigungsfeld immer größer. Ganz neue Chancen liegen in den interdisziplinären Ansätzen. Nehmen wir als Beispiel das automatisierte und vernetzte Fahren. Stetig sind neue und verbesserte Assistenzsysteme in das Automobil eingezogen. Vom ABS bis zum heute zumindest begrenzt stattfindenden autonomen Fahren ist eine Lawine an neuen Funktionen über uns hereingebrochen. Digitalisierung und künstliche Intelligenz sind nicht mehr wegzudenken. Mit den Automatisierungsstufen 0 bis 5 versuchen Institutionen wie SAE, BAST und VDA, Ordnung ins System zu bringen. Ziele sind, das Fahren sicherer zu machen und die Schädigung der Umwelt gering zu halten. Gleichzeitig geht es um die besten Produkte und Arbeitsplätze, sprich die Sicherung unseres Wohlstands. Das Auto wird neu erfunden und wir reden über die Mobilitätswende. Die Begeisterung der Ingenieure kennt keine Grenzen.

Aber halt! Selbst wenn die Assistenz- und Automatisierungssysteme tatsächlich wie angepriesen arbeiten und wenn wir Themen wie funktionale Sicherheit und Security in den Griff bekommen, muss doch gefragt werden: „Wo bleibt der Mensch?". Mensch und Technik ist zwar kein wirklich neues
Prof. Dr. Hans-Christian Reuss Leiter des Lehrstuhls für Kraftfahrzeugmechatronik an der Universität Stuttgart und Mitglied des Vorstands des Forschungsinstituts für Kraftfahrwesen und Fahrzeugmotoren Stuttgart (FKFS)
Thema. Ergonomen und Psychologen sind eingebunden, und wir machen viele Probandenstudien in Fahrsimulatoren, um die Akzeptanz der Funktionen zu überprüfen. Aber genügt das? Müssen wir nicht vollständig umdenken? Wir sollten den Spieß umdrehen und statt immer neue Produkte mit neuen Funktionen auf den Markt zu bringen, intensiver die Frage stellen, was die Bedürfnisse des Menschen eigentlich sind. Davon ausgehend sollten wir die Anforderungen an neue Produkte ableiten. Das wäre nicht nur nachhaltiger, sondern wir würden auf Menschen stoßen, die im Lauf ihres Lebens die Fähigkeiten ihrer Sinnesorgane einbüßen und bei denen die Fähigkeiten in Bezug auf Denk- und Bewegungsvermögen schwinden. Wir würden feststellen, dass der Anteil der „Alten“ zunimmt. „Da wir immer älter werden, erleben wir auch in zunehmendem Maß, dass unsere Organe schlechter werden, denn diese sind für ein so hohes Lebensalter entwicklungsgeschichtlich gar nicht konzipiert", sagt Ulrich Schiefer, der als Augenarzt sowohl im Studiengang Augenoptik an der Hochschule Aalen als auch an der Universitäts-Augenklinik Tübingen arbeitet.

Der eNOVA-Strategiekreis Automobile Zukunft hat den Meilenstein 2035 seiner Forschungs-Roadmap wie folgt überschrieben: „Integrierte, nachhaltige und nutzerfreundliche Mobilität durch intelligentes, nahtloses, automatisiertes Zusammenwirken von Fahrzeug, Infrastruktur und Mensch“. Ich bin dankbar, dass hier der Mensch ganz neu in den Mittelpunkt gerückt wird und plädiere für „Technik für Menschen“ statt „Mensch der Technik“. 